\title{
Evaluating SOA Nonlinearity Effects by High Order Susceptibility on Constant Envelope Signals in WDM Systems
}

\author{
Silvello Betti, and Elisa Duca
}

\begin{abstract}
Nonlinearity effects, arising within a semiconductor optical amplifier, have been deeply investigated with particular interest for exploiting them in wavelength conversion. This application shrinks the research area to a precise scenario: a strong pump signal travels through the active medium simultaneously with a modulated signal to be converted. In this case it is possible to neglect the spectral distribution of the optical power and phase relationship between propagating signals. SOAs have been also studied as line amplifiers for WDM phase modulated signals. Aim of this paper is to extend the propagation model in a SOA, in order to simulate nonlinearities occurring in the amplification of a WDM comb signal evaluating FWM on constant envelope modulation schemes (DPSK). This can be carried out thanks to an enanched analytical model based on the optical field and medium interaction, overcoming the wide-used Rate Equation approach, which is based on the overall photons density.
\end{abstract}

Index Terms-Susceptibility,Semiconductor Optical Amplifier (SOA), Four Wave Mixing (FWM), Cross Gain Modulation (XGM), Cross Phase Modulation (XPM), Nonlinear Effects, Nonlinear Crosstalk, Differential Phase Shift Keying (DPSK), Wavelength Division Multiplexing (WDM).

\section{INTRODUCTION}

$\mathbf{N}$ ON-LINEAR phenomena induced by saturated Semiconductor Optical Amplifiers (SOAs), have been widely investigated for their effects on amplitude-modulated signals [1]. Most of these phenomena can be successfully exploited for wavelength conversion [2], regeneration [3], optical gating [4], and demultiplexing [5]. Many effects, as Cross Gain Modulation (XGM), have reference to free carriers modulation. XGM arises from the depletion of available carriers inside the active medium, for the presence of a high power optical pulse: if other signals are simultaneously propagating through the amplifier, they undergo a reduced gain as long as the available carriers number is reduced. This is one of the main impairments for developing SOA based amplification in WDM amplitude modulated systems [6]. There are other causes for carrier modulation: for example, the propagation of a $\mathrm{CW}$ comb induces a carrier modulation whose pulsation is related to the frequency spacing. Any change in the refractive index of the active medium is transposed into a phase modulation of the optical field. There are several sources behind the phase variations induced on a constant envelope signal from a SOA: Self Phase Modulation (SPM), Cross Phase Modulation (XPM) and Four Wave Mixing (FWM). In order to provide a simulative model for multichannel amplification in SOAs,

S. Betti and E. Duca are with the Department of Electrical Engineering, Tor Vergata University, Rome, 00133 Italy, e-mail: \{betti,duca\}@ing.uniroma2.it these mechanisms must be taken into account. The most implemented models, are based on Rate Equations, which allow to calculate the time and space variation of free carrier and photons densities inside the active medium. This approach is widely used and provides very good results in case of single channel amplification, or when a strong signal propagates with another probe signal, for example to exploit XGM and perform wavelength conversion. It requires several coefficients, whose values are set as constant in most of the cases: linear, differential and compression gain coefficients and linewidth enhancement factor [7]. In some applications, these coefficients are not allowed to be constant, and a dynamic calculation must be run. Since these terms come from the medium susceptibility, it is worth to calculate the susceptibility and from that derive the optical field evolution in the active medium [8], [9]. This is not an alternative approach with respect to Rate Equations, it calculates the exact functions from whom all coefficients used in Rate Equations come out. In this paper we will give a close expression for the complete susceptibility, up to its third order taking into account all nonlinear phenomena such as Self Phase Modulation, Cross Gain Modulation and Four Wave Mixing in case of multiple channels propagation. We are being concerned with nonlinear effects on constant envelope WDM signal simultaneously amplified by a SOA, since this is a scenario where the Rate Equations based models limitations are mostly evident. Further details on comparison between susceptibility based and Rate Equations based approaches will be given in a dedicated section. Results will be eventually described: we are considering constant envelope phase modulated signals, in order to highlight the phase impairments instead of amplitude related phenomena, as XGM. Given the unknown statistics behind the phase impairments, qualitative but accurate results may come from the $Q_{\text {eye }}$ factor [10].

\section{NONLINEAR PROPAGATION OF MULTIWAVELENGTH} SIGNAL IN A SEMICONDUCTOR TWO-LEVELS SYSTEM

By applying Maxwell Equations to a nonlinear and nonmagnetic medium, the $k_{t h}$ spectral component for the electric field, propagating along $z$ is [11]:

$$
-\nabla^{2} A_{k}(z, t)+\frac{\omega_{k}^{2} n^{2}}{c^{2}} \cdot A_{k}(z, t)=-\frac{1}{\varepsilon_{0} c} \frac{\partial^{2} P_{k}}{\partial t^{2}}
$$

where $A_{k}$ and $P_{k}$ are the complex envelope of the electric field and the polarization, slowly varying with respect to the optical carrier. In order to get a close solution for $A_{k}$, an expression 
for $P_{k}$ is required [12]. Aim of this section is to find an expression of the polarization in a semiconductor active medium when it undergoes an interaction with an electromagnetic field. Matter-field interaction and all the consequent phenomena are well described by the density matrix formalism [13]-[15]. This approach models the semiconductor active medium as a two level system, the density matrix is reduced to a $2 \times 2$ matrix, with elements $\rho_{c c}, \rho_{v c}, \rho_{c v}$ and $\rho_{v v}$. The energy levels correspond to the conduction and valence bands. Thanks to this formalism, it is easy to calculate the occupation probabilities for each band and the transition probability.

We assume each signal frequency component to be independent, with a narrow bandwidth with respect to the spectral spacing; i.e. we are considering $10 \mathrm{Gbit} / \mathrm{s}$ data rate, in order to avoid any spectral overlapping with adjacent channels, which may occur for phase modulated signals when the spectral spacing is $50 \mathrm{GHz}$. Of course if the channel spacing is set to any higher value (100 or $200 \mathrm{GHz})$ the data rate can be increased. We associate a couple of energy state $\left(\mathcal{E}_{c, v}\right)$ to a resonance frequency $(\zeta)$. Each resonance frequency collects the energy transitions between the conduction and valence band, and vice-versa.Each signal is supposed to have a narrow bandwidth, in order to associate a resonance frequency to every single optical carrier of the WDM system. If signals have a wide bandwidth this hypothesis is no longer satisfied, and the density matrix approach can not be applied.

The next step is to calculate the interaction between the optical field and the carrier density for each energy level, as well as the carrier transition probability from one state to another. The polarization, which is related to interaction processes between light and matter, can be calculated from the off diagonal density matrix elements $\rho_{c v}$ and $\rho_{v c}$. Its scalar component can be found from:

$$
\mathcal{P}=\frac{1}{V} \sum_{\kappa} \mu_{\kappa}\left[\rho_{c v, \kappa}+\rho_{v c, \kappa}\right],
$$

where $\mu_{\kappa}$ is the electric dipole [13], associated to a transition at wavenumber $\kappa$. It is possible to derive an expression for the off-diagonal elements of the density matrix, which are related to an energy transition between the conduction band $\left(\mathcal{E}_{c}\right)$ and the valence band $\left(\mathcal{E}_{v}\right)$, whose energy distance can be written as: $\hbar \zeta$. The density matrix operator is directly related to the Hamiltonian of the system [12]. After some steps, we get:

$$
\begin{aligned}
\frac{d \rho_{v c}}{d t} & =(i \zeta-\gamma) \rho_{v c}+i \frac{\mu}{\hbar} \mathcal{E}\left(\bar{\rho}_{c c}-\bar{\rho}_{v v}\right), \\
\frac{d \rho_{c v}}{d t} & =-(i \zeta+\gamma) \rho_{c v}-i \frac{\mu}{\hbar} \mathcal{E}\left(\bar{\rho}_{c c}-\bar{\rho}_{v v}\right), \\
\frac{d \rho_{v v}}{d t} & =-\gamma_{v}\left(\rho_{v v}-\bar{\rho}_{v v}\right)-\frac{i \mu}{\hbar} \mathcal{E}\left(\rho_{c v}-\rho_{v c}\right)+\Lambda_{v}, \\
\frac{d \rho_{c c}}{d t} & =-\gamma_{c}\left(\rho_{c c}-\bar{\rho}_{c c}\right)-\frac{i \mu}{\hbar} \mathcal{E}\left(\rho_{c v}-\rho_{v c}\right)+\Lambda_{c},
\end{aligned}
$$

note that $\rho_{c v}=\rho_{v c}^{*}$. In Eq. 5 and 6 we introduced $\Lambda_{c, v}$ as the conduction band and valence band electrons variation for electric pumping. Previous equations include the collision terms, referred to the 'spin-spin' relaxation time $T_{2}=1 / \gamma$, for the inter-band transition, $\tau_{c}$ and $\tau_{v}$ are the intraband energy relaxation time, for conduction and valence band, respectively. From a physical point of view, $\rho_{c c}$ and $\rho_{v v}$ represent the occupation probability for the conduction and the valence band state, respectively. We introduced $\bar{\rho}_{c c}$ and $\bar{\rho}_{v v}$ as the occupation probability in absence of an optical field. The variation induced by an optical field is separable from the thermal equilibrium contribution:

$$
\rho_{c c}=\bar{\rho}_{c c}+\Delta \rho_{c c}(0)+\sum_{k \neq 0} \Delta \rho_{c c}(k \Omega) e^{-i k \Omega t},
$$

where we added to the thermal equilibrium a nonlinear contribution $\Delta \rho_{c c}$. The occupation probability changes with the spectral spacing of the optical field components: $\Omega$ represents the detuning between two adjacent channels: $\Omega=\omega_{k+1}-\omega_{k}$; we recall that the static change in the occupation probability $\Delta \rho_{c c}(0)$ is referred to the static hole burning, on the other hand the beating between two modes makes a modulation arise at the detuning pulsation generating the so-called phenomenon of population pulsation [16]. The same formalism is adopted for describing the valence band occupation probability. The off-diagonal elements, on the other hand, are solvable after the introduction of the following formalism:

$$
\rho_{v c}(t)=\sum_{k}\left[\bar{\rho}_{v c}\left(\omega_{k}\right)+\Delta \rho_{v c}\left(\omega_{k}\right)\right] e^{-i \omega_{k} t},
$$

which is a complex function. Once all elements for the matrix are found, it is possible to solve Eq.2 and calculate the polarization. It is worth highlighting that the resonance pulsation $\zeta$ is defined over a set of discrete elements, according to the wavevector $\kappa$. In particular, the time evolution for the density matrix and its elements are defined over the wavevector values, which identify the quantum states for each energetic band. There will be transitions from one band to another, only for the same wavevector. Thus, a density matrix realization is defined for each wavenumber. The electrical dipole $\mu$ has been introduced, without stressing its dependence on the pulsation. As a matter of fact, it is a function of the energy of the carriers involved in the transition. A detailed description of the dipole function is presented in [17] and [18], where the following expression is reported:

$$
|\mu(\zeta)|^{2}=\frac{q^{2}}{6 m_{0} \zeta^{2}}\left(\frac{m_{0}}{m_{c}}-1\right) \frac{\mathcal{E}_{g}\left(\mathcal{E}_{g}+\Delta_{0}\right)}{\mathcal{E}_{g}+2 \Delta_{0} / 3},
$$

where $m_{c}$ is the carriers mass, $m_{0}$ is the effective mass, $q$ is the electron charge, $\mathcal{E}_{g}$ is the energy gap, and $\Delta_{0}$ is the spin-orbit splitting. The solution of differential equations (Eq. 3-6), for the matrix elements, is strongly influenced by the optical field. We distinguish between different frequencies considered: $\omega_{k}$ is the channel carrier, $\zeta$ is related to the transition energy from conduction to valence band. This second frequency does not depend on the signal optical carrier or bandwidth, but on the device active medium: doping, temperature and geometry can change the spectral shape of the transition function. In other words, this second frequency depends on the Fermi levels, and 
the current. By deriving the Eq.8, we get:

$$
\begin{aligned}
\dot{\rho}_{c v}(t)= & \sum_{k=1}^{M}\left(-i \omega_{k}\right)\left(\bar{\rho}_{c v}+\Delta \rho_{c v}\left(\omega_{k}\right)\right) e^{-i \omega_{k} t}+ \\
& \sum_{k=1}^{M} \frac{d}{d t}\left(\bar{\rho}_{c v}+\frac{d \Delta \rho_{c v}}{d t}\left(\omega_{k}\right)\right) e^{-i \omega_{k} t}
\end{aligned}
$$

By substituting Eq.10 and Eq.8 into Eq.4, assuming that both $\bar{\rho}_{c v}$ and $\Delta \rho_{c v}(t)$ are slower than the exponential terms, we have:

$$
\begin{aligned}
& \sum_{k=1}^{M}\left(-i \omega_{k}\right)\left[\bar{\rho}_{c v}\left(\omega_{k}, t\right)+\Delta \rho_{c v}\left(\omega_{k}, t\right)\right] e^{-i \omega_{k} t}+ \\
& \left(\frac{1}{T_{2}}-i \zeta\right)\left[\bar{\rho}_{c v}\left(\omega_{k}, t\right)+\Delta \rho_{c v}\left(\omega_{k}, t\right)\right] e^{-i \omega_{k} t}= \\
& -\frac{\mu}{i \hbar}\left(\bar{\rho}_{v v}-\bar{\rho}_{c v}\right)\left(\sum_{j=1}^{M} A_{j} e^{-i \omega_{j} t}+A_{j}^{*} e^{i \omega_{j} t}\right)+ \\
& -\frac{\mu}{i \hbar}\left(\sum_{K \neq 0}\left(\Delta \rho_{v v}(K \Omega)-\Delta \rho_{c c}(K \Omega)\right) e^{-i K \Omega t}\right) \times \\
& \left(\sum_{j=1}^{M} A_{j} e^{-i \omega_{j} t}+A_{j}^{*} e^{i \omega_{j} t}\right),
\end{aligned}
$$

where we have introduced $K \Omega=\omega_{m}-\omega_{n}$. In the first step, $\Delta \rho_{c v}$ is neglected and it will be taken into account for the higher order terms. The solution is:

$$
\bar{\rho}_{c v}\left(\omega_{k}\right)=\frac{\mu}{\hbar} \frac{A_{k}\left(\bar{\rho}_{c c}-\bar{\rho}_{v v}\right)}{\omega_{k}-\zeta+i \gamma}, \quad \bar{\rho}_{v c}\left(\omega_{k}\right)=\bar{\rho}_{c v}\left(\omega_{k}\right)^{*}
$$

Once the time-independent term of the off-diagonal elements are found, we proceed with the calculation of higher orders conduction (and valence) band occupation probabilities, for which the derivation leads to:

$$
\dot{\rho}_{c c}(t)=\sum_{k=1}^{M}\left((-i k \Omega) \Delta \rho_{c c}(k \Omega)+\frac{d \Delta \rho_{c c}}{d t}(k \Omega)\right) e^{-i k \Omega t} .
$$

This expression is to be substituted into Eq.6, where the offdiagonal elements are required. By substituting and simplifying we have:

$$
\begin{aligned}
\Delta \rho_{c c}(0) & =\frac{2 \mu^{2} \gamma \tau_{c}}{\hbar^{2}}\left(\bar{\rho}_{v v}-\bar{\rho}_{c c}\right) \sum_{k} \frac{\left|A_{k}\right|^{2}}{\gamma^{2}+\left(\zeta-\omega_{k}\right)^{2}} \\
\Delta \rho_{v v}(0) & =\frac{2 \mu^{2} \gamma \tau_{v}}{\hbar^{2}}\left(\bar{\rho}_{c c}-\bar{\rho}_{v v}\right) \sum_{k} \frac{\left|A_{k}\right|^{2}}{\gamma^{2}+\left(\zeta-\omega_{k}\right)^{2}}
\end{aligned}
$$

The complete expression for the conduction band occupation probability requires the terms responsible of population pulsation, which comes from the beating between different optical carriers:

$$
\begin{gathered}
\Delta \rho_{c c}\left(\omega_{m}-\omega_{n}\right)=\frac{i \mu^{2}\left(\bar{\rho}_{c c}-\bar{\rho}_{v v}\right)}{\hbar^{2}\left(\gamma_{c}-i\left(\omega_{m}-\omega_{n}\right)\right)} \times \\
\left(\frac{1}{\omega_{m}-\zeta-i \gamma}-\frac{1}{\omega_{n}-\zeta+i \gamma}\right) A_{m} A_{n}^{*}
\end{gathered}
$$

Now we have all contributions required to calculate $\Delta \rho_{v c}$ by solving Eq.11. This time the exponential terms introduce a constrain on the possible interaction between optical carriers. We must introduce the hypothesis $\omega_{k}=\omega_{j}+\omega_{m}-\omega_{n}$, in order to guarantee the phase matching condition and simplify the exponential terms. Thus:

$$
\begin{aligned}
& \Delta \rho_{c v}\left(\omega_{k}\right)=-\frac{\mu^{3}\left(\bar{\rho}_{c c}-\bar{\rho}_{v v}\right)}{\hbar^{3}\left(\omega_{k}-\zeta+i \gamma\right)} \times \\
& \sum_{k} \sum_{m} \sum_{n} A_{m}(z, t) A_{n}^{*}(z, t) A_{j}(z, t) \times \\
& \left(\frac{1}{\omega_{m}-\omega_{n}+i \gamma_{c}}+\frac{1}{\omega_{m}-\omega_{n}+i \gamma_{v}}\right) \times \\
& \left(\frac{1}{\omega_{m}-\zeta-i \gamma}-\frac{1}{\omega_{n}-\zeta+i \gamma}\right) .
\end{aligned}
$$

With this last expression, we can find the polarization by solving Eq. 2 with the formalism introduced in Eq.11, both the contributions are now available from Eq.12 and Eq.17. Then, the susceptibility can be derived. In particular, from $\rho_{c v}$ and $\Delta \rho_{c v}$ several contributions to high order susceptibility may be distinguished. The linear susceptibility $\chi_{L}$ comes from Eq.12, on the other hand, all the nonlinear terms due to Kerr phenomenon are collected in Eq.17. Thus, the overall susceptibility has the following expression:

$$
\begin{aligned}
\bar{\chi}\left(\omega_{k}\right) & =\chi_{L}\left(\omega_{k}\right)+\chi_{S P M}\left(\omega_{k} ; \omega_{k},-\omega_{k}, \omega_{k}\right) \\
& +\sum_{m \neq k} \chi_{X P M}\left(\omega_{k} ; \omega_{m},-\omega_{m}, \omega_{k}\right) \\
& +\sum_{m} \sum_{n \neq m} \sum_{j} \chi_{F W M}\left(\omega_{k} ; \omega_{m},-\omega_{n}, \omega_{j}\right),
\end{aligned}
$$

thus the slowly varying polarization can be written as:

$$
P_{k}(t)=\bar{\chi}\left(\omega_{k}, t\right) A_{k}(t) .
$$

The sum in Eq.2 can be changed into an integration, in a continuous domain, by exploiting the relationship between the wavenumber $\kappa$ and the pulsation $\zeta$. In order to perform a change of variables on the integration domain, the characterization of the conduction and valence band profile in terms of Energy versus wavenumber, is required. This step introduces new terms collected in the so-called 'density function' [19]. The density function $(f(\zeta))$ also gathers common terms in Eq.12 and Eq.17. For a bulk SOA $f(\zeta)$ has the following expression:

$$
f\left(\zeta, \omega_{k}\right)=\left(\frac{2 m_{c} m_{v}}{m_{c}+m_{v}}\right)^{3 / 2} \frac{\sqrt{\hbar \zeta-E_{g}}}{\omega_{k}-\zeta+i \gamma}\left(\bar{\rho}_{c c}-\bar{\rho}_{v v}\right),
$$

where $m_{c}$ and $m_{v}$ are the carriers and holes effective masses [20], $E_{g}$ the energy gap calculated as a function of the carrier density [19], $\gamma=1 / T_{2}$ with $T_{2}$ the dipole relaxation time, $\bar{\rho}_{c c}$ and $\bar{\rho}_{v v}$ are the electron and hole occupation probabilities at steady conditions. Their values are calculable with the Fermi distribution functions [18]:

$$
\bar{\rho}_{x x}=\frac{1}{e^{\frac{\hbar \zeta-E_{f_{x}}}{k T}}+1}
$$

where the quasi Fermi levels $E_{f_{x}}(x=c, v)$ are considered, $k$ is the Boltzmann constant and $T$ is the system temperature. The quasi Fermi levels are functions of the available carriers in each energy state. An estimation of the quasi Fermi levels 
is given in [21], and widely used in several simulative models [19].The linear contribution to the susceptibility can be written in a closed form:

$$
\chi_{L}\left(\omega_{k}, t\right)=\int_{E_{g} / \hbar}^{\infty} \frac{\mu^{2}(\zeta)}{\varepsilon_{0} \hbar} f\left(\zeta, \omega_{k}\right) d \zeta,
$$

on the other hand, the third term of the susceptibility can be derived in a simplified form as:

$$
\begin{aligned}
& \chi_{S P M}\left(\omega_{k}, t\right)= \int_{E_{g} / \hbar}^{\infty}-\frac{\mu^{4}(\zeta)}{\varepsilon_{0} \hbar^{3}} f\left(\zeta, \omega_{k}\right) \bar{\gamma} \frac{2 i \gamma\left|A_{k}\right|^{2}}{\left(\omega_{k}-\zeta\right)^{2}+\gamma^{2}} d \zeta \\
& \chi_{X P M}\left(\omega_{k}, t\right)= \int_{E_{g} / \hbar}^{\infty}-\frac{\mu^{4}(\zeta)}{\varepsilon_{0} \hbar^{3}} f\left(\zeta, \omega_{k}\right) \bar{\gamma} \sum_{m \neq k} \frac{2 i \gamma\left|A_{m}\right|^{2} d \zeta}{\left(\omega_{m}-\zeta\right)^{2}+\gamma^{2}} \\
& \chi_{F W M}\left(\omega_{k}, t\right)= \int_{E_{g} / \hbar}^{\infty}-\frac{\mu^{4}(\zeta)}{\varepsilon_{0} \hbar^{3} f\left(\zeta, \omega_{k}\right) \sum_{j} \sum_{m} \sum_{n \neq m} \frac{A_{m} A_{j} A_{n}^{*}}{A_{k}} .} \\
&\left(\frac{1}{\omega_{m}-\omega_{n}+i \gamma_{v}}+\frac{1}{\omega_{m}-\omega_{n}+i \gamma_{c}}\right) \times \\
&\left(\frac{1}{\omega_{m}-\zeta+i \gamma}-\frac{1}{\omega_{n}-\zeta-i \gamma}\right) d \zeta .
\end{aligned}
$$

In Eq.24 we introduced $\bar{\gamma}=-i\left(\gamma_{c}+\gamma_{v}\right) /\left(\gamma_{v} \cdot \gamma_{c}\right)$. From Eq.25 it is clear that not only the single channel optical power counts for the higher order processes, but also the complex envelope of the optical field. Moreover, Eq.24 is referred to XPM, as well as to XGM phenomena. In particular, the real and the imaginary parts of the susceptibility have their effects on the phase and the amplitude of the optical field, respectively. Thanks to its complete expression, the susceptibility allows to evaluate phase phenomena arising from the simultaneous propagation of several optical carriers. The optical phase is not calculated as in rate-equation based models, where the phase variation comes from a static parameter known as the linewidth enhancement factor [22]. Its theoretical derivation comes directly from the susceptibility but it is commonly used as a constant for calculating the optical dephasing. By including the susceptibility, the phase of each optical carrier can see dynamically not only the overall optical power fluctuations, but also how this power is spectrally distributed. All the contributions to the susceptibility are complex, and can be directly related to the nonlinear Schrödinger Equation.

\section{CARRIER DENSITY DYNAMICS}

The interaction between the media and the traveling optical field can be completed by the definition of the carrier density variation. The carrier is required to calculate each susceptibility term, by the $f\left(\zeta, \omega_{k}\right)$ function, which depends directly on the carrier density. Indeed, this pulsation is one of the most important reasons for FWM to arise [18]. In such a scenario the carrier density can not be found notwithstanding what so far introduced. In particular, the carrier density variation is [23]:

$$
\dot{N}(t)=\frac{I}{q V}-\frac{N(t)}{\tau_{s}}-\frac{i \varepsilon}{4 \hbar}\left[\mathbf{P}^{*} \mathbf{E}-\mathbf{P} \mathbf{E}^{*}\right]
$$

where the carrier lifetime $\tau_{s}^{-1}=A+B N(t)+C N^{2}(t)$. The solution, according to the phase matching conditions, is:

$$
N(t)=\frac{I \tau_{s}}{q V}+\frac{i \varepsilon}{4 \hbar} \sum_{k, j} \frac{\bar{\chi}^{*}\left(\omega_{k}, t\right)-\bar{\chi}\left(\omega_{j}, t\right)}{\frac{1}{\tau_{s}}-j\left(\omega_{j}-\omega_{k}\right)} A_{k}^{*} A_{j} e^{-i\left(\omega_{j}-\omega_{k}\right) t}
$$

where we used the notation $\bar{\chi}=\bar{\chi}_{R}+j \bar{\chi}_{I}$, numerically evaluated as a function of the carrier density. At first sight, Eq. 27 contains the explicit expression of carrier modulation. In this equation, the carrier density does not depend simply on the photon density, but on the electric field complex envelopes, the medium susceptibility, and the spectral spacing of the WDM channels. When a set of modulated channels are injected into the SOA, the carrier density expression requires some more details in order to guarantee higher accuracy. By comparing Eq.27 with the common used one in rate equation based models [24], there is an important difference: we do neglect nor the optical field spectral components, neither the optical phase of each carrier. Thus, the spectral spacing of the optical field has an effect on the carrier density variation, even if none of the signals is amplitude modulated. This means interchannel crosstalk is a non-negligible phenomenon when the channel spacing is narrow, or the overall optical power is higher than the saturation power. The rate equation model does not consider the spectral distribution of the optical power, but the mean power is considered by neglecting spectral spacing even if two optical signals are propagating. This simplification suits when the system to be modeled foresees a strong pump signal and a modulated signal, i.e. in wavelength conversion technique. In that case, the optical power difference is too high and the spectral spacing is too wide to appreciate the modulation induced by population pulsation. There are several scenarios, this simplification holds no longer good. In the following sections we will consider a WDM system with DPSK signalling. The constant envelope of DPSK would apparently lead to any carrier modulation. As a matter of fact, the population pulsation arises for several reasons, and leads to impairments able to reduce the system performance in particular conditions.

\section{COMPARISON With RATE-EQUATIONS BASED MODELS}

The set of rate equations is hereafter reported:

$$
\begin{aligned}
& \frac{d N(t)}{d t}=\frac{I(t)}{q}-\frac{N(t)}{\tau_{n}}-g \frac{N(t)-N_{0}}{1+\varepsilon S_{t o t}(t)} S(t) \\
& \frac{d S(t)}{d t}=\left(g \frac{N(t)-N_{0}}{1+\varepsilon S_{t o t}(t)}-\frac{1}{\tau_{p}}\right) S(t)+\beta \frac{N(t)}{\tau_{n}} \\
& \frac{d \varphi(t)}{d t}=\frac{\alpha}{2}(N(t)-\bar{N})
\end{aligned}
$$

When it comes to multi-wavelength signal propagation, Rate Equations must be written taking into account the spectral distribution of photons. One the one hand, the photon equation must be written for each channel, in order to take into account the signal modulation and adequate gain coefficients; on the other hand, the overall photons $S_{t o t}$ should be expressed as a function of the overall optical power:

$$
P_{t o t} \propto\left|E_{t o t}\right|^{2}=\sum_{i}\left|E_{i}\right|^{2}+E_{i} \cdot \sum_{j \neq i} E_{j}^{*} .
$$


The beating terms introduce a modulation of the optical power, whose frequency is the spectral spacing between each channel pair. The photon rate equation must inherit this term, with a proper weight on each wavelength participating the beating, depending on the gain spectral shape. As a matter of fact, when beating phenomenon between channels arises, each one suffers a modulation due to a local perturbation of the carrier density and thef refractive index, which is not constant in frequency. Thus, the overall free carrier density suffers a wide-band modulation and, consequently, all phenomena related to the refractive index variation are affected by an inter-channel cross-talk. This phenomenon is well described through Eq.27, where each pair of channels participating in the beating process are taken into account with their own time varying susceptibility. From that equation, it is possible to relate the gain coefficient $g$ in Eq. 28 to the imaginary part of the susceptibility. When a single channel propagation is considered, that term can be simplified into a constant coefficient. Otherwise a spectral shape is considered when wavelength conversion is modeled, and a differential gain coefficient is introduced. In both the cases Rate Equations guarantee good fitting and simple computation, this makes the rate-equations model attractive. When interchannel cross-talk is no longer negligible, this simplification is sometimes not possible. When the active medium gain has to be calculated in the time domain, the susceptibility must be used. Moreover, the gain compression coefficient $\varepsilon$, is also derivable from the susceptibility. It is related to a reduction of $\chi_{L}$ when the optical power increases, since $\chi_{S P M}, \chi_{X G M}$ and $\chi_{F W M}$ are subtracted from the linear term. Thus, the more power propagates the lower material gain is available. This approach allows to take into account all sources for gain compression, which may arise from the spectral spacing, from data pattern (cross-talk), and instantaneous optical power distributed over all the channels. The same can be said for the linewidth enhancement factor $\alpha$ in Eq.30. That term is set as constant, but it is well known that its expression is based on the susceptibility variation with respect to the carrier density:

$$
\alpha=\frac{\partial \bar{\chi}_{R} / \partial N}{\partial \bar{\chi}_{I} / \partial N} .
$$

This remarks that the rate equation models are based on the susceptibility, too. When the reference scenario foresees multichannel propagation of phase modulated signal, the linewidth enhancement factor should be updated in time and calculated for each frequency. This step requires the knowledge of susceptibility. Thus, it may be easier to calculate the optical field propagation via the susceptibility itself without passing through the photon density in rate equations.

\section{APPLICATION TO A WDM-DPSK COMMUNICATION SYSTEM}

In order to highlight the nonlinear phase noise, we consider a DPSK signalling communication systems with NRZ pulse shape. This to reduce the gain modulation induced by amplitude modulation, and to emphasize the nonlinear phase impairments from channels interaction. Phase impairments

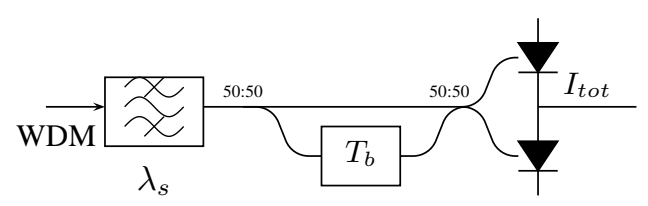

Fig. 1. Receiver scheme for a DPSK signalling. The WDM is filtered in order to select one optical carrier, then the interferometer demodulates the signal in the optical domain. A complete conversion of signal format is given by the balanced receiver, which, in addition, reduces the noise level in the received electrical signal.

TABLE I

SOA'S MAIN PARAMETERS USED IN SIMULATIONS

\begin{tabular}{|c||c|c|}
\hline Parameter & Value & Unit \\
\hline$L$ & 300 & $\mu m$ \\
$V$ & $6.5 \times 10^{-17}$ & $\mathrm{~m}^{3}$ \\
$m_{r}$ & $3.772 \times 10^{-32}$ & $\mathrm{Kg}$ \\
$T_{2}$ & 50 & $f s$ \\
$\tau_{c}$ & 0.65 & $p s$ \\
$\tau_{v}$ & 0.2 & $p s$ \\
$A$ & $10^{8}$ & $s^{-1}$ \\
$B$ & $2 \times 10^{-16}$ & $s^{-1} \cdot m^{-3}$ \\
$C$ & $2 \times 10^{-41}$ & $s^{-1} \cdot m^{-6}$ \\
$T_{b}$ & 100 & $p s$ \\
$I$ & 26 & $m A$ \\
\hline
\end{tabular}

can be evaluated, by means of the third order susceptibility which takes into account all phenomena generating signal dephasing. The receiver is based on an interferometric demodulator and balanced photodetectors configuration [25]. In this work no additive noise due to Amplified Spontaneous Emission (ASE) is taken into account at the receiver end: the signal is assumed to be noise free, in order to highlight the impairments coming from nonlinear crosstalk. Each channel carries a phase modulated, constant envelope signal. The set of channels is injected into the amplifier, where it undergoes amplification under nonlinear conditions. After a single stage amplification, each channel is filtered and converted into the electrical domain, using the receiver scheme reported in Figure 1. The current resulting from the photodetection process in a balanced receiver is proportional to the cosine of the differential phase shift, thus all the phase noises induced on the optical field are transposed into a current variation. Thanks to the high order susceptibility, these phase variations are appreciable even in case of constant envelope signals. Eventually, the electrical signal is filtered and the BER is calculated after the integration-and-dump operation. Simulations were carried out for several overall optical power values. We considered four simultaneously propagating signals, among which the overall optical power is equally distributed. Different spectral spacing has been considered: 50, 100 and $200 \mathrm{GHz}$. This to emphasize the best and worst operative conditions for WDM signalling to be amplified by a SOA without previously be filtered. We considered a $300 \mu \mathrm{m}$ long SOA, with detailed parameters reported in Tab. I [18]. In order to evaluate the crosstalk impairments due to multichannel propagation, the main parameter to consider is the overall optical power, that 
may move the amplifier operative conditions from linear into the saturation regime.

\section{A. Results from Simulations and Discussions}

Numerical solutions for the susceptibility and the traveling optical field, were found by developing a code which implements the following algorithm. The amplifier is divided into longitudinal slices, whose length is $d z=v_{g} * d t$ where $v_{g}$ is the group velocity inside the active medium assumed to be constant, $d t$ is the sampling time for the incoming optical signal. Inside each slice of the active medium, an interaction between the available carrier and the traveling field is considered. This local interaction leads to a spatial distribution of the available carrier. We aim to calculate the optical field in each section. Each wavelength suffers the instantaneous spectral distribution of several other optical carriers. Thus, the susceptibility is required. Once the free available carrier density is obtained, the actual susceptibility value is computable for each wavelength, and the optical phase and amplitude variations can be determined. The effect of nonlinear coupling between channels is evaluated for different amplifier parameters, and different optical incoming power values. In particular, for a given amplifier, the increase of the overall optical power leads to a stronger nonlinear interaction, since the amplifier works in its saturation regime. On the other hand, for a given optical power, the same saturation regime is reached by changing the amplifier parameters. We placed the channels in correspondence of the left slope of the amplifier gain, thus, the higher the wavelength the higher the gain it undergoes. The effects on each channel have been evaluated by the Q-factor.Even if we deal with non gaussian noise, this parameter can be used to give a qualitative trend of the impairments [26]-[28]. We calculated the Q-factor from the empirical mean values and standard deviations on the electrical received signal. We do not go any further with the BER calculation, since that step requires more considerations on the statistical nature of the noises. The Q-factor is calculated from the electrical received signal, from an interferometer with balanced receiver. Signals at lower wavelengths, corresponding to lower optical gain region, exhibit an increasing $Q$, thus better performances, with the overall optical power. On the other hand, signals with higher gain perform worse with the increase of the overall optical power. It is worth to recall that Q-factor only takes into account amplitude phenomena. Thus, the optical phase noise introduced by nonlinear crosstalk, can be hidden by a strong gain. For this reason we are analyzing the Q-factor in this section, but in the following one more accurate calculation on the optical de-phasing $(\Delta \varphi)$ will be given. We consider four channels with several spectral spacing: 50,100 and $200 \mathrm{GHz}$. It is interesting to compare the effects of : amplifier saturation, amplifier gain tilt, and spectral spacing on each signal performance. Actually it is hard to distinguish between these phenomena, because the larger spectral spacing brings the signal at higher wavelength to higher gain. Thus, each signal suffers the gain any other signal undergoes. If this gain increases, the nonlinear interaction changes. The signals at lower wavelength seem to be improved by the increase of

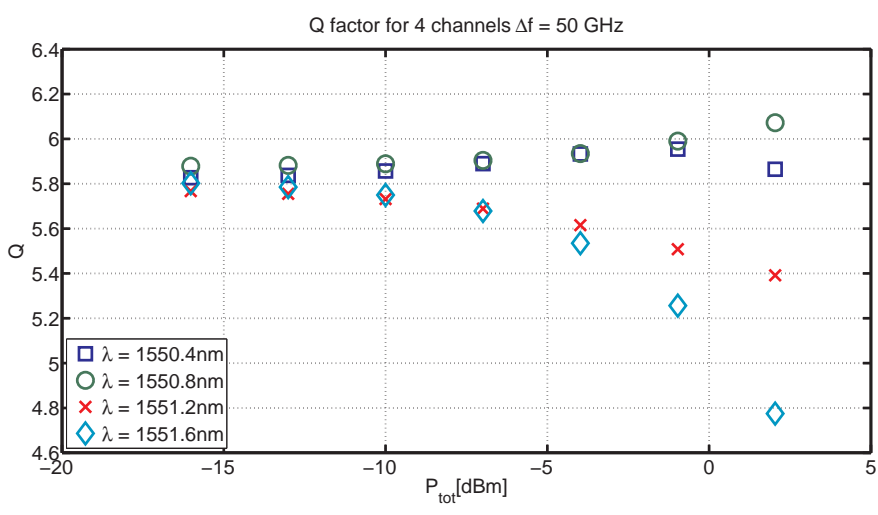

Fig. 2. Q factor for four channels spectrally spaced of $50 \mathrm{GHz}$.

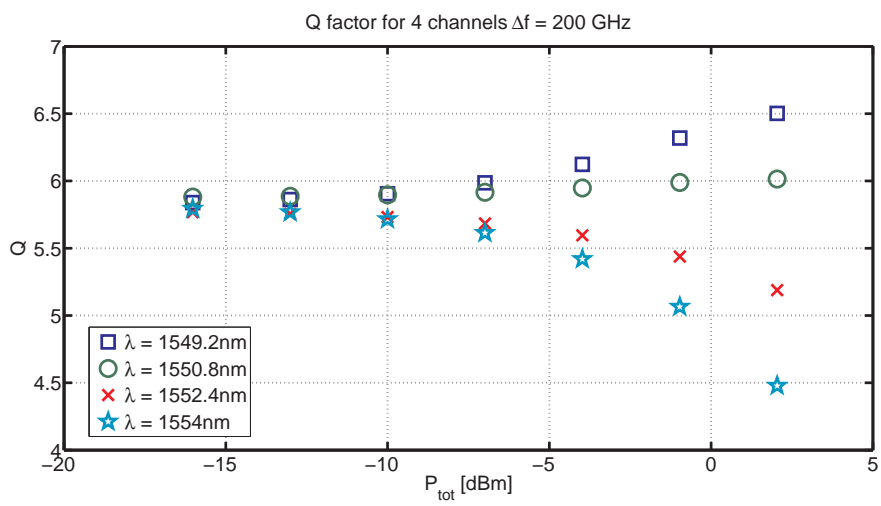

Fig. 3. Q factor for four channels spectrally spaced of $200 \mathrm{GHz}$.

the optical power. The opposite happens to signals at higher wavelengths. In Figure 2 and Figure 3 results for four channels propagation are reported.

It is possible to appreciate a lower dynamics for $\mathrm{Q}$ factor in case of narrow spectral spacing. If the optical power increases, the peak gain is shifted to higher wavelength, for a reduction of the available free carriers. The relative reduction of the gain, is one of the reasons which induce changes on the $\mathrm{Q}$ factor. When the spectral spacing is wider, this effects is even more appreciable. In this case, indeed, effects related to gain tilt phenomena are more relevant, since a wider spectral spacing reduces the correlation between channels relatively to gain spectral profile related processes. In order to highlight the effects due to phase nonlinearities on the $\mathrm{Q}$ factor of a single channel, more results are reported.We isolated a single channel $(\lambda=1551.6 \mathrm{~nm})$, in case of 8 propagating channels. We consider the effect of the spacing and the optical power on that channel. As it is shown in Figure 4, there is no relevant effect in propagation if the overall optical power is low. With the increase of the optical power, the spectral spacing influences the system performances in terms of $\mathrm{Q}$ factor. In particular, narrower the spacing, lower the optical power which drives the system out of service. In our case a spacing of $100 \mathrm{GHz}$ has a margin of about $4 \mathrm{~dB}$ on the allowed optical power with respect of a spacing of $50 \mathrm{GHz}$. 


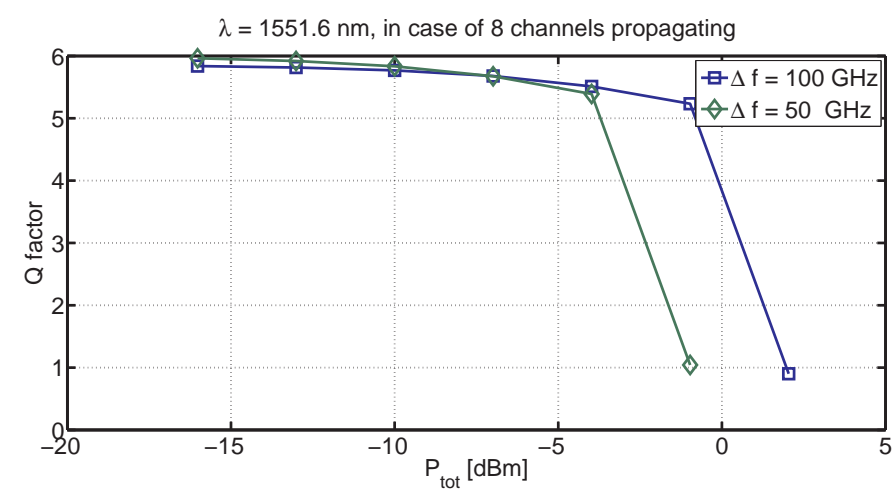

Fig. 4. Q factor for $\lambda=1551.6 \mathrm{~nm}$ when eight signals are simultaneously propagating, with different spectral spacing.

\section{PhASE IMPAIRMENTS}

With our approach it is possible to calculate the optical phase impariments induced by the semiconductor on each carrier. Thus, it is possible to isolate the phase noise in the optical domain which is no longer appreciable in the electrical domain, after the photodetection. As a matter of fact, all phase changes are translated into an amplitude electrical noise and it is not distinguishable from other amplitude noises. The optical phase is influenced by the real part of the global susceptibility calculated in Eq.18, which takes into account also the nonlinear interaction between the propagating optical carriers, which leads to nonlinear cross-talk. All these phase impairment sources are collected in the following expression:

$$
\begin{aligned}
\Delta \varphi\left(\omega_{k}, t\right)= & \Re\left\{j \frac { 2 \pi } { c \omega _ { k } } \int _ { 0 } ^ { L } \left(\bar{\chi}\left(\omega_{k}, t, z^{\prime}\right) \omega_{k}^{2}+\right.\right. \\
& \left.\left.2 j \omega_{k} \dot{\bar{\chi}}\left(\omega_{k}, t, z^{\prime}\right)-\ddot{\bar{\chi}}\left(\omega_{k}, t, z^{\prime}\right)\right) d z^{\prime}\right\},
\end{aligned}
$$

which comes from the solution of Eq.1, where the polarization is substituted with its expression based on susceptibility. The proposed equation allows us to find a numerical value for the phase impairment on each WDM channel. This approach is not based on a simple coefficient which may not take into account the spectral position of the channel and the presence of other channels. The equation is based on the calculation of the susceptibility derived in Eq. from 23 to 25 . We calculate the standard deviation and the mean value for the optical induced dephasing $(\Delta \varphi)$, from their ratio we obtain an indication on the dependances of the optical phase noise and nonlinear crosstalk. In particular, as it is shown in Figure 5, the inner channels suffer a higher phase penalties than the border channels do, and exhibit lower Q-factor. It must be remarked that in our simulations we allocated all channels on the left slope of the optical gain, thus the lowest wavelength channel undergoes a smaller gain than the highest wavelength channel does. For a narrow spectral spacing the inner channels suffer a stronger de-phasing than the border channels. But when the spectral spacing increases, FWM becomes negligible, and, in addition, the gain gap between channels is the main source of phase variation. Starting from these considerations it is possible to give a qualitative indication on the trend of optical de-phasing induced by the nonlinear crosstalk, even

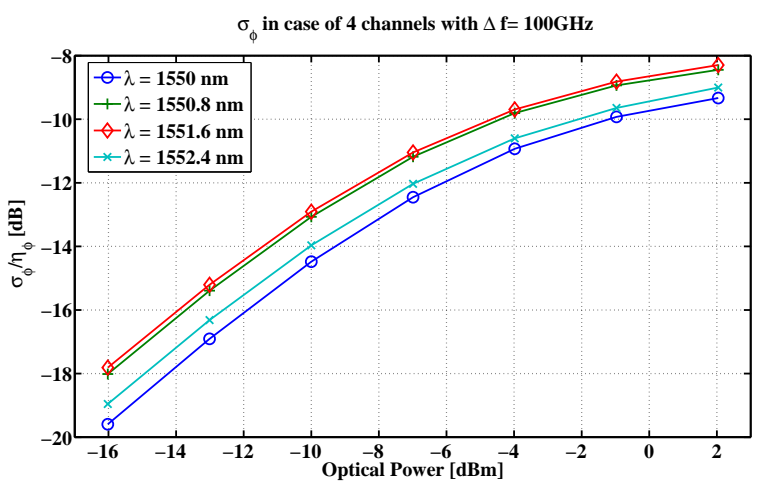

Fig. 5. Phase noise standard deviation, in case of four channels propagating.

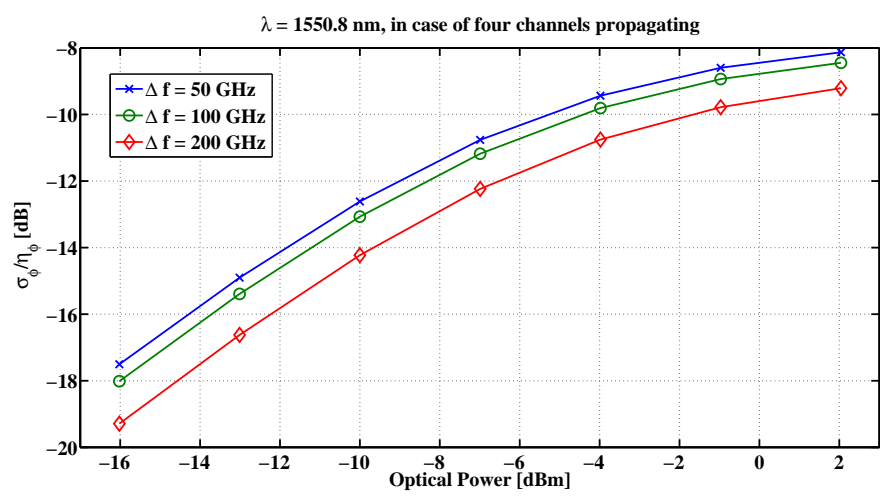

Fig. 6. Phase noise standard deviation, in case of four channels propagating.

on constant envelope signals. What the Q-factor could hide is more evident with the calculation of the optical phase shift: higher optical power leads to stronger de-phasing, and inner channels are the most penalized ones from that process. Of course the global system performance must be calculated via the Q-factor in the electrical domain, but this parameter collects all the impairments bringing also phase processes into the amplitude of the electrical signal.

\section{FURTHER APPLICATIONS}

The proposed approach overcomes the Rate Equation based model, giving more accurate results in terms of field propagation. Interesting results may come from the introduction of the additive noise, or ASE noise. This would lead to a consistent comparison of the BER penalty induced by nonlinear crosstalk and the BER penalty induced by the optical noise. In addition the saturation regime is modified by the presence of ASE noise, and the linear regime is shrunk for high ASE levels. Finally, by introducing ASE generated by SOA under investigation it will be possible to evaluate the phase noise in case of low OSNR. In that case the nonlinear phase noise is a relevant effect on system performance. Moreover, we recall we carried out this subject assuming a constant envelope signals, as DPSK. Stronger results may come from not-constant envelope signals, as OOK, since nonlinear phenomena would be stressed. Moreover, the receiver configuration may change the SNR by manipulating the optical signals and noises. 


\section{CONCLUSIONS}

In this paper we have used the density matrix approach to model the SOA, evaluating the effects of WDM-DPSK signalling propagation inside the active medium. The optical field has been considered instead of the photon density, which does not give any information on the phase noise and the FWM process arising in the active medium. We showed some results on the simultaneous propagation of constant envelope signals in a SOA: nonlinear processes arising are not negligible, and may introduce considerable impairments if not properly treated. The impairments have been calculated via the Q-factor for several signals configurations, in terms of optical power and spectral spacing. Eventually, a measurement of the optical phase noise was given.

\section{ACKNOWLEDGMENTS}

The authors would like to thank Silvia Di Bartolo and Davide Massimiliano Forin for helpfull discussions and for carrying out preliminary experimental trials.

\section{REFERENCES}

[1] A. Reale, S. D. Carlo, P. Lugli, D. Campi, C. Cacciatore, A. Stano, and G. Fornuto, "Study of gain compression mechanisms in multiplequantum-well $\operatorname{In}_{1-x} \mathrm{Ga}_{x}$ As semiconductor optical amplifiers," IEEE Journal of Quantum Electronics, vol. 35, pp. 1697-1703, Nov. 1999.

[2] G. Contestabile, N. Calabretta, M. Presi, and E. Ciaramella, "Single and multicast wavelength conversion at $40 \mathrm{gb} / \mathrm{s}$ by means of fast nonlinear polarization switching in an SOA," IEEE Photonics Technology Letters, vol. 17 , no. 12 , pp. 2652-2654, Dec. 2005.

[3] H. Simos, A. Bogris, and D. Syvridis, "Investigation of a $2 \mathrm{R}$ all-optical regenerator based on four-wave mixing in a semiconductor optical amplifier," IEEE.

[4] G. Berrettini, A. Simi, A. Malacarne, A. Bogoni, and L. Potì, "Ultrafast integrable and reconfigurable XNOR, AND, NOR, and NOT photonic logic gate," IEEE Photonics Technology Letters, vol. 18, no. 8, pp. $917-$ 919, Apr 2006.

[5] C. Schubert, S. Diez, J. Berger, R. Ludwig, U. Feiste, H. G. Weber, G. Toptchiyski, K. Petermann, and V. Krajinovic, "160-gb/s all-optical demultiplexing using a gain-transparent ultrafast-nonlinear interferometer (GT-UNI)," IEEE Photonics Technology Letters, vol. 13, no. 5, pp. 475-477, May 2001.

[6] E. Ciaramella, A. D'Errico, and V. Donzella, "Using semiconductor optical-amplifiers with constant envelope WDM signals," IEEE Journal of Quantum Electronics, vol. 44, pp. 403-409, May 2008.

[7] I. Fatadin, D. Ives, and M. Wicks, "Numerical simulation of intensity and phase noise from extracted parameters for cw dfb lasers," IEEE Journal of Quantum Electronics, vol. 42, no. 9, pp. 934-941, Sep. 2006.

[8] S. Balle, "Simple analytical approximations for the gain and refractive index spectra in quantum-well lasers," Phys. Rev. A, vol. 57, no. 2, pp. 1304-1312, Feb 1998.

[9] J. Revuelta, L. Pesquera, and S. Balle, "Wavelength-dependent output properties of quantum well optical amplifiers," Conference on Laser and Electro-Optics, pp. 420-421, 2000.

[10] G. Bosco and P. Poggiolini, "On the Q factor inaccuracy in the performance analysis of optical direct-detection dpsk systems," IEEE Photonics Technology Letters, vol. 16, no. 2, pp. 665-667, Feb. 2004.

[11] G. Agrawal, Nonlinear fiber optics, ser. Optics and Photonics. San Diego, CA USA: Academic Press, 2001.

[12] R. Kazarinov, C. Henry, and R. Logan, "Longitudinal mode selfstabilization in semiconductor lasers," J. Appl. Phys., vol. 53, pp. 46314644, Jul 1982.

[13] A. Yariv, Quantum Electronics, 3rd ed. John Wiley \& Sons, 1989.

[14] B. Thedrez, A. Jones, and R. Frey, "Two-level description of gain and mixing susceptibility in amplifying semiconductor materials," IEEE Journal of Quantum Electronics, vol. 24, pp. 1499-1506, Aug. 1988.

[15] D. Hjelme and A. Mickelson, "Gain nonlinearities due to carrier density dependent dispersion in semiconductor lasers," IEEE Journal of Quantum Electronics, vol. 25, pp. 1625-1631, Jul. 1989.
[16] G. Agrawal, "Gain nonlinearities in semiconductor lasers: Theory and application to distributed feedback lasers," IEEE Journal of Quantum Electronics, vol. 23, no. 6, pp. 860-868, 1987.

[17] M. Asada, A. Kameyama, and Y. Suematsu, "Gain and intervalence band absorption in quantum-well lasers," IEEE J. Quantum Electron., vol. QE-20, pp. 745-753, Jul 1984.

[18] A. Uskov, J. Mörk, and J. Mark, "Wave mixing in semiconductor laser amplifiers due to carrier heating and spectral-hole burning," IEEE Journal of Quantum Electronics, vol. 30, no. 8, pp. 1769-1781, Aug. 1994.

[19] M. Connelly, Semiconductor Optical Amplifiers. Dordrecht, The Netherlands: Kuwler Academic Publishers, 2002.

[20] A. Matsumoto, K. Nishimura, K. Utaka, and M. Usami, "Operational design on high-speed semiconductor optical amplifier with assist light for application to wavelength converters using cross-phase modulation," IEEE Journal of Quantum Electronics, vol. 42, pp. 313-323, mar 2006.

[21] N. Nilsson, "Empirical approximation for the fermi energy of a semiconductor with parabolic bands," Appl. Phys. Lett., vol. 33, pp. 653-654, 1978.

[22] N. Storkfelt, B. Mikkelsen, D. Olesen, M. Yamaguchi, and K. Stubkjaer, "Measurement of carrier lifetime and linewidth enhancement factor for $1.5 \mu \mathrm{m}$ ridge waveguide laser amplifier," IEEE Photonic Technology Letters, vol. 3, pp. 632-634, Jul 1991.

[23] C. Ning, R. Indik, and J. Moloney, "Effective bloch equations for semiconductor lasers and amplifiers," IEEE Journal of Quantum Electronics, vol. 33, pp. 1543-1550, Sep. 1997.

[24] A. Reale, S. D. Carlo, and P. Lugli, "Gain dynamics in traveling-wave optical amplifiers," IEEE Journal of on Selected Topics in Quantum Electronics, vol. 07, no. 2, pp. 293-299, 2001.

[25] K.-P. Ho, Phase-Modulated Optical Communication Systems. NY USA: Springer Science+Business Media Inc., 2005.

[26] F. Matera and M. Settembre, "Comparison of the performance of optically amplified transmission systems," IEEE Journal of Lightwave Technology, vol. 14, pp. 1-12, Jan 1996.

[27] A. Mecozzi, "Limits to long-haul coherent transmission set by the kerr nonlinearity and noise of the in-line amplifiers," IEEE Journal of Lightwave Technology, vol. 12, pp. 1993-2000, Nov 1994.

[28] N. S. Bergano, F. Kerfoot, and C. Davidson, "Margin measurements in optical amplifier systems," IEEE Photonics Technology Letters, vol. 5, pp. 304-306, Mar 1993.

Silvello Betti Silvello Betti received the Laurea degree 'cum laude' in Electrical Engineering from the University "La Sapienza" of Rome, Italy, in 1984. In 1985 he was granted a fellowship at Fondazione Ugo Bordoni, Rome, on a joint project with Alcatel-Face Research Center, Pomezia (Italy), concerning on networking applications of optical coherent systems. In 1986 he joined Fondazione Ugo Bordoni, where he was engaged with the Optical Communication Group as a researcher on coherent optical systems and their applications in point-to-point trunks, LAN, MAN and in intersatellite optical links. Since 1995 he has been working on optic fibre nonlinear propagation, access optical networks and hybrid fibre-radio systems. Since 1990-91 he has been teaching Optical Fibres and Integrated Optics in Optics and Optoelectronics Specialization Lectures for Italian military forces (FF.AA.). In 1998 he joined the University of L'Aquila (Italy), where he was Associate Professor of Electrical Communications. In 2001 he joined the University of Rome "Tor Vergata" (Italy), where he is Associate Professor of Optical Communication Systems and Signal Theory. He is co-author of a book on optical fibre communications (S. Betti, G. De Marchis, E. Iannone, Coherent Optical Communications Systems, Wiley-Interscience, New York, 1995) and he is author or co-author of more than 120 papers published in international journals and presented in international conferences

Elisa Duca Elisa Duca graduated 'cum laude' in 2002 at the University of Rome 'Tor Vergata', where she finished her PhD in Telecommunications and Microelectronics Engineering in 2005. From 2008 she is researcher at the Italian Space Agency, working on optical intersatellite links. Her research areas involve optical intersatellite links as well as fiber links, semiconductor optical amplifiers modeling, fiber nonlinearities, and Fiber Bragg Gratings for sensing applications. 